\title{
Improved KLT Algorithm for High-Precision Wavelength Tracking of Optical Fiber Bragg Grating Sensors
}

\author{
Daniele Tosi \\ School of Engineering, Nazarbayev University, 53 Kabanbay Batyr, Astana 010000, Kazakhstan \\ Correspondence should be addressed to Daniele Tosi; daniele.tosi@nu.edu.kz
}

Received 10 August 2016; Accepted 11 October 2016; Published 18 January 2017

Academic Editor: Banshi D. Gupta

Copyright ( 2017 Daniele Tosi. This is an open access article distributed under the Creative Commons Attribution License, which permits unrestricted use, distribution, and reproduction in any medium, provided the original work is properly cited.

\begin{abstract}
Fiber Bragg Gratings (FBGs) are among the most popular optical fiber sensors. FBGs are well suited for direct detection of temperature and strain and can be functionalized for pressure, humidity, and refractive index sensing. Commercial setups for FBG interrogation are based on white-light sources and spectrometer detectors, which are capable of decoding the spectrum of an FBG array. Low-cost spectrometers record the spectrum on a coarse wavelength grid (typically $78-156$ pm), whereas wavelength shifts of $1 \mathrm{pm}$ or lower are required by most of the applications. Several algorithms have been presented for detection of small wavelength shift, even with coarse wavelength sampling; most notably, the Karhunen-Loeve Transform (KLT) was demonstrated. In this paper, an improved algorithm based on KLT is proposed, which is capable of further expanding the performances. Simulations show that, reproducing a commercial spectrometer with $156 \mathrm{pm}$ grid, the algorithm estimates wavelength shift with accuracy well below $1 \mathrm{pm}$. In typical signal-to-noise ratio (SNR) conditions, the root mean square error is $22-220 \mathrm{fm}$, while the accuracy is $0.22 \mathrm{pm}$, despite the coarse sampling. Results have been also validated through experimental characterization. The proposed method allows achieving exceptional accuracy in wavelength tracking, beating the picometer level resolution proposed in most commercial and research software, and, due to fast operation $(>5 \mathrm{kHz})$, is compatible also with structural health monitoring and acoustics.
\end{abstract}

\section{Introduction}

Established through the last decade as a prominent sensing technology, Fiber Bragg Gratings (FBGs) are gaining popularity as a sensing technology [1-3]. FBGs combine the advantages of fiber-optic sensors (small size, lightweight, immunity to electromagnetic interference, and biocompatibility) with specific features, which make them attractive for several industrial applications. FBGs are low-cost devices with a straightforward demodulation principle and allow the realization of wide and compact sensor networks, exploiting both time- and wavelength-division multiplexing (TDM/WDM) [3].

FBGs are compact and reflective optical filters, inscribed within an optical fiber, which reflect a single wavelength [4, 5]. Each FBG is sensitive to strain and temperature, exhibiting a linear sensitivity over a wide sensing range [2]. FBG strain sensors are employed in structural health monitoring $[6,7]$, avionics [8,9], vibration sensing [10-13], and seismic applications $[14,15]$; FBGs are also often embedded into composite materials [6]. Miniature FBG sensors are often employed in medicine for temperature $[16,17]$ and strain sensing [18-20] and in harsh environments such as nuclear plants [21, 22]. FBG sensors have also been functionalized to pressure [20], magnetic [23,24], and chemical [25] detection, usually with the help of transductors.

The latest research efforts are increasing the potential of FBGs, as a sensing approach. Improving over the traditional phase mask fabrication $[26,27]$, the possibility of inscribing FBGs with draw-tower approach $[28,29]$ and with femtosecond lasers [30] is providing a strong expansion of the FBG capabilities. Furthermore, FBGs are combining with multicore fibers [31] and with shape-sensing approaches [32], for additional sensing capabilities.

Over the last years, commercial interrogators have significantly improved their affordability, consolidating the 
white-light principle [1]. In this arrangement, a broadband source is used to illuminate the array of FBG sensors, and a spectrometer or a spectrum analyzer is used to collect the reflection spectra, determining the wavelength shift of each FBG. In order to further reduce costs, inexpensive spectrometers can be used as detectors [33]; however, the penalty is that spectrometers sample wavelength usually over 512 pixels over a bandwidth of $40-80 \mathrm{~nm}$, therefore achieving a wavelength resolution insufficient for a direct spectral detection. Thus, it is necessary to develop an algorithm capable of discriminating small wavelength shifts, in spite of a coarse spectral sampling, in order to functionally integrate any type of FBG sensor into a high-precision measurement system.

Several detection algorithms have been documented, for tracking FBG wavelength shifts in a white-light setup. The simplest approaches are curve fitting [34, 35], centroid detection [36], and FBG bandwidth detection; these algorithms are often used on the software of commercial instruments [33, 37-39]. The spectrum correlation method, proposed in $[40,41]$, is extremely reliable to low signal-tonoise ratio (SNR) conditions, but it is limited by the narrow wavelength grid sampling. Recently, the method proposed by Lamberti et al. [42] based on fast phase correlation makes a significant step forward and achieves an accuracy of 7-35 fm with $10 \mathrm{pm}$ wavelength grid. Other approaches based on discrete wavelet transform have also been presented [43].

Recently, a demodulation technique for FBG sensors based on Karhunen-Loeve Transform (KLT) $[44,45]$ was proposed for the first time. The technique works by analyzing the Fourier transform of the FBG spectrum, coarsely sampled, and further processing it by determining its eigenvalues. The kernel of the detection method is the identification of the highest rank eigenvalue, which finds its roots into the method proposed by Maccone [46-48] for detection of carrier signal buried into noise.

In this paper, the KLT algorithm for detection is revised and improved, through the analysis of the whole eigenvalue set, with the goal of improving the algorithm in two areas: (1) sound trade-off between accuracy and resilience to SNR, obtaining a subpicometer accuracy for standard SNR values (40-65 dB in most systems) and with a good resilience to SNR worsening; (2) computation time, having a decoding algorithm compatible for real-time detection at the maximum speed of the spectrometer (typically $1-5 \mathrm{kHz}$ ). The paper will show, both in an accurate simulative context and in experimental validation, that simple modifications to the KLT core [44] will achieve the task.

The paper is arranged as follows. Section 2 reviews the principle of operation and white-light setup-based interrogation of FBG sensors and sets the premises for the simulation benchmark for the performance analysis. Section 3 describes the KLT decoding method, in its original form and with the proposed improvements. Section 4 describes the obtained results, emphasizing in particular the accuracy and SNR resilience. Section 5 provides a brief experimental validation in dynamic and static sensing. Finally, Section 6 draws conclusions.

\section{Fiber Bragg Gratings: Principles and Interrogation}

2.1. FBG Principles. An FBG is a periodic modulation of the refractive index of an optical fiber [1-4]. This is typically fabricated by means of an ultraviolet light excitation and modulated by a diffractive phase mask [26], although more recent techniques based on draw-tower inscription [28] and femtosecond laser inscription [30] have been proposed. For a uniform fiber grating, we assume that the modulation period $\Lambda$ and the effective refractive index of the fiber core $n_{\text {eff }}$ are both constant along the grating direction. In this case, Erdogan's coupled mode theory provides a closed-form expression for the FBG spectrum [4]. In particular, the FBG behaves as a narrow-band reflective filter, centered at the socalled Bragg wavelength $\lambda_{B, 0}$ :

$$
\lambda_{B, 0}=2 n_{\mathrm{eff}} \Lambda,
$$

where the subscript 0 refers to the absence of any temperature or strain variation. The reflection spectrum of an FBG can be approximated as $[2,4]$

$$
R(\lambda)=\frac{\sinh ^{2}\left(L \sqrt{\kappa^{2}-\sigma^{2}}\right)}{\cosh ^{2}\left(L \sqrt{\kappa^{2}-\sigma^{2}}\right)-\sigma^{2} / \kappa^{2}},
$$

where $\lambda$ is the wavelength; $R(\lambda)$ is the reflectivity; $L$ is the grating length. $\kappa L$ is the grating strength parameter, which is a unitless number that typically ranges from 0.1 for weak grating to 4 for strong grating. The maximum grating reflectivity is $\tanh ^{2}(\kappa L)$. If $\delta n_{\mathrm{eff}}$ is the amplitude of the refractive index modulation, in reference conditions $\left(\lambda_{B}=\right.$ $\left.\lambda_{B, 0}\right), \sigma$ has the following expression $[2,4]$ :

$$
\sigma=\frac{\pi}{\lambda} \delta n_{\mathrm{eff}}+2 \pi n_{\mathrm{eff}}\left(\frac{1}{\lambda}-\frac{1}{\lambda_{B}}\right) .
$$

Equations (1)-(3) provide a framework for simulating an FBG in reference conditions, when no variations of strain or temperature are applied to the grating. The FBG, however, is sensitive to both strain and temperature, as both $\Lambda$ and $n_{\text {eff }}$ depend on their variations. According to [2, 3], for small variations of temperature $\Delta T$ and strain $\varepsilon$, we observe a linear shift $\Delta \lambda$ of the Bragg wavelength $\lambda_{B}$ :

$$
\begin{aligned}
\Delta \lambda= & 2\left(\Lambda \frac{\partial n_{\mathrm{eff}}}{\partial \varepsilon}+n_{\mathrm{eff}} \frac{\partial \Lambda}{\partial \varepsilon}\right) \varepsilon \\
& +2\left(\Lambda \frac{\partial n_{\mathrm{eff}}}{\partial \Delta T}+n_{\mathrm{eff}} \frac{\partial \Lambda}{\partial \Delta T}\right) \Delta T .
\end{aligned}
$$

Equation (4) is typically reformatted as

$$
\Delta \lambda=k_{\varepsilon} \varepsilon+k_{T} \Delta T
$$

highlighting the strain coefficient $k_{\varepsilon}$ and the temperature coefficient $k_{T}$. For FBGs operating in the third optical window and based on standard or photosensitive fibers, typical parameters are $k_{\varepsilon}=1 \mathrm{pm} / \mu \varepsilon$ and $k_{T}=10 \mathrm{pm} /{ }^{\circ} \mathrm{C}$ [3]. Thus, 


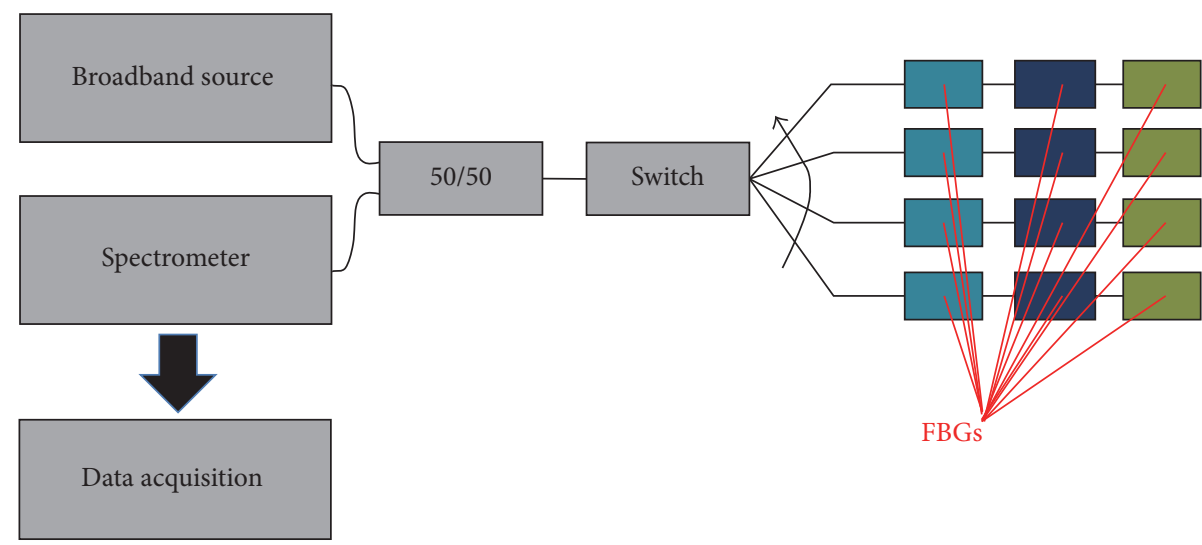

FIGURE 1: Schematic of a generic FBG interrogation based on white-light setup.

in presence of strain and/or temperature variations, the Bragg wavelength $\lambda_{B}$ in (1) and (3) shifts by the quantity $\Delta \lambda$ :

$$
\lambda_{B}(\varepsilon, \Delta T)=\lambda_{B, 0}+\Delta \lambda(\varepsilon, \Delta T)=\lambda_{B, 0}+k_{\varepsilon} \varepsilon+k_{T} \Delta T .
$$

Equations (1)-(6) provide the basis for FBG sensors: thermal or mechanical stimuli applied to the FBG result in a linear shift of the FBG spectrum; this model is remarkably accurate for small variations, whereas the linear relationship is preserved and no spectral distortions are introduced. Our goal is to estimate the smallest values of wavelength shift $\Delta \lambda$, in order to be able to detect the smallest variations of the sensing parameter.

2.2. Interrogation. Most FBG interrogators, based on commercial devices or self-assembled in research laboratories, are based on a white-light setup as in Figure 1. A broadband source typically a superluminescent light emitting diode (SLED) coupled into an optical fiber is used as a light source. A 3-dB coupler, or a circulator, is used to decouple the backreflection component. One or more FBGs, all having different wavelengths, form an array of sensors operating in WDM; eventually, an optical switch can be used to commute between different channels. The backreflection component is decoded with a fiber-coupled spectrometer.

In order to make FBG sensing attractive and affordable, it is desirable to operate with inexpensive and compact detectors [33]. Typically, CCD-based spectrometers used in fiber-optic sensors have a working bandwidth of $40-80 \mathrm{~nm}$ and return the optical spectrum $R(\lambda)$ discretized over both the wavelength (horizontal axis) and the amplitude (vertical axis).

Spectrometers sample the wavelength with 9-bit (512 pixel) resolution, in most of the cases. This leads to a typical wavelength resolution of $78 \mathrm{pm}$ or $156 \mathrm{pm}$, which is significantly inferior to optical spectrum analyzer (OSA) instruments that achieve a resolution bandwidth of 5-20 pm. On the other side, spectrometers have lower cost, over one order of magnitude lower than OSAs, have compact size and low power consumption, as they are usually powered via the USB port of a computer, and have faster response, up to $1-5 \mathrm{kHz}$ using high-speed data transfer protocols such as
RS-232 [33, 38]. The spectral amplitude is quantized over 16 bits (65536 values), for the vast majority of spectrometers. The 9-bit wavelength and 16-bit amplitude discretization are parameters common to most commercial spectrometers [33] and FBG analyzers [38, 39] based on white-light setup.

In the following simulations, we refer to the worst-case scenario, underlying the following hypotheses.

(i) The wavelength axis is quantized over 512 values, from $1520 \mathrm{~nm}$ to $1600 \mathrm{~nm}$, as in [33, 44]. Hence, the wavelength grid is sampled with a resolution of $156 \mathrm{pm}$.

(ii) The amplitude axis is quantized over 32768 values, half of the available range of operation, in order to introduce a "safety margin" that avoids spectral saturation in case of power fluctuations.

(iii) We neglect the neighboring effect, between different FBGs composing the array. In other words, we assume that the spectral spacing between adjacent FBGs is sufficient to guarantee negligible interference. Thus, we can reduce the performance analysis to a single FBG and extend the results to all FBGs composing the array. This hypothesis is well realistic when it applies to small thermal/strain variations of an FBG, which is the case of interest for this paper, whereas variations will be limited to $\pm 5 \mathrm{pm}$.

(iv) The reflection spectrum, prior to be quantized, is perturbed by additive white noise (AWN). The AWN provides in this case a worst-case scenario, because unlike $1 / f$ and high-frequency noise (which are often present in spectrometers), it cannot be filtered through a whitening or adaptive filter [49].

(v) The SNR figure is defined as the ratio between the variance of the useful signal (i.e., the reflection spectrum of the FBG) and the noise variance.

(vi) The spectrum of the optical source is normalized.

(vii) The parameters chosen for simulation are $L=5 \mathrm{~mm}$, $\kappa L=1, \delta n_{\text {eff }}=10^{-5}$, and $n_{\text {eff }}=1.5$. The reference wavelength is chosen as $\lambda_{B, 0}=1550 \mathrm{~nm}$. 


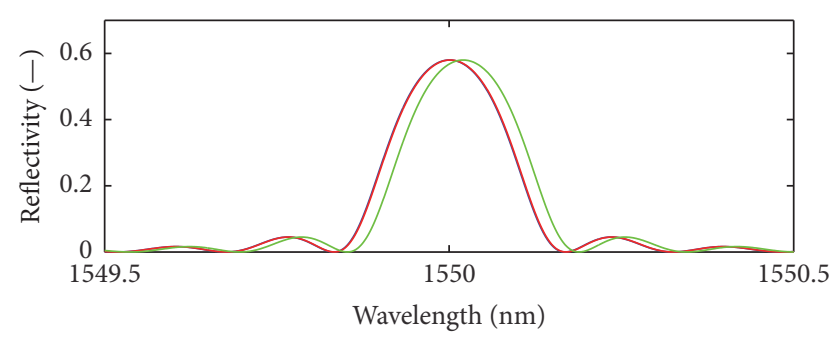

(a)

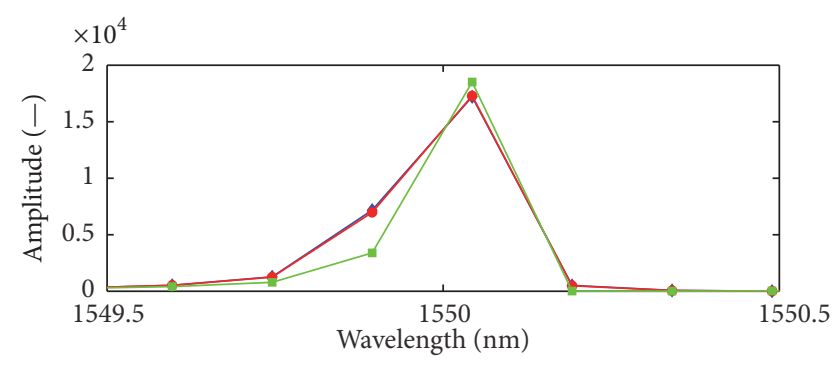

$$
\begin{aligned}
\rightarrow \Delta \lambda & =0 \mathrm{pm} \\
\because \Delta \lambda & =1 \mathrm{pm} \\
-\Delta \lambda & =20 \mathrm{pm}
\end{aligned}
$$

(b)

Figure 2: Spectrum of an FBG centered at $1550 \mathrm{~nm}$, in absence of noise, generated by means of coupled mode theory [4], for different values of spectral shift $(0 \mathrm{pm}, 1 \mathrm{pm}$, and $20 \mathrm{pm})$. (a) shows all spectral details, with 1 pm quantization, in ideal conditions. (b) shows the spectra after quantization on both axes, as visualized on a typical spectrometer.

In these conditions, Figure 2 shows the spectrum of a uniform FBG in reference conditions and with $1 \mathrm{pm}$ and $20 \mathrm{pm}$ wavelength shift, prior and after quantization. It is possible to see that the quantization process, even in absence of noise, clears all the spectral details that most algorithms [34-36, 42] make use for wavelength shift estimation. The entire FBG spectrum is then contained within the 6 spectral samples, and the majority of the spectral information is encoded in 4 samples. In this condition, we need to use a detection algorithm that is effectively capable of converting small variations of amplitude into significant variations of its output.

\section{Wavelength Detection Algorithm}

3.1. Original KLT Algorithm. The wavelength detection procedure is an algorithm that applies to the decoded spectrum, after the inner portion correspondent to the FBG has been isolated. We assume that the wavelength axis $\underline{\lambda}=$ $\left[\lambda_{1}, \lambda_{2}, \ldots, \lambda_{N}\right]$ is discretized, and so is the amplitude axis of the reflection spectrum $\underline{R}(\underline{\lambda})=\left[R\left(\lambda_{1}\right), R\left(\lambda_{2}\right), \ldots, R\left(\lambda_{N}\right)\right]=$ $\left[R_{1}, R_{2}, \ldots, R_{N}\right]$. This way, we will operate only on digital signals. In the following procedure, the length of digital signals is $N=14$.

As in [44], the first step is to process the array $\underline{R}$ with a Fast Fourier Transform (FFT) procedure:

$$
\underline{G}=\left[G_{1}, \ldots, G_{N}\right]=\operatorname{FFT}(\underline{R})
$$

whereas each sample $G_{k}(k=1, \ldots, N)$ of the array $\underline{G}$ is computed with the classical method:

$$
G_{k}=\sum_{n=1}^{N} R_{n} e^{j 2 \pi k(n / N)} .
$$

Subsequently, the array $\underline{G}$ is expanded into its Toeplitz matrix $M$ :

$$
\underline{\underline{M}}=\left[\begin{array}{ccccc}
G_{1} & G_{2} & G_{3} & \cdots & G_{N} \\
G_{2} & G_{1} & G_{2} & \ddots & \vdots \\
G_{3} & G_{2} & G_{1} & \ddots & G_{3} \\
\vdots & \ddots & \ddots & \ddots & G_{2} \\
G_{N} & \cdots & G_{3} & G_{2} & G_{1}
\end{array}\right] .
$$

Then, we apply the KLT to the matrix $\underline{M}$, by finding an orthonormal basis that can decompose the matrix into its fundamental components. As shown by Maccone [46], this operation can be computationally performed through the singular value decomposition (SVD) that decomposes the matrix into its eigenvalues and eigenvectors [44]:

$$
\underline{\underline{M}}=\underline{\underline{V}} \times \underline{\underline{D}} \times \underline{\underline{V}}^{-1}
$$

where $\underline{D}$ is a diagonal matrix, containing all the $N$ eigenvalues on the main diagonal and $\underline{V}$ is a full matrix, containing on each $k$ th column the eigenvector corresponding to the $k$ th eigenvalue. Computationally, the SVD is implemented with the Cholesky decomposition, which is the default operator in commercial processing software, such as MATLAB and LabVIEW.

Calling $\xi$ the eigenvalue string, containing the $N$ diagonal elements of $\underline{D}$, we assume that all eigenvalues are ranked in ascending order:

$$
\underline{\xi}=\left[\left|\xi_{1}\right|<\left|\xi_{2}\right|<\cdots<\left|\xi_{N}\right|\right]
$$

we note that all eigenvalues are real numbers since $M$ is symmetric, and negative eigenvalues tend to appear only when the SNR is very low. 


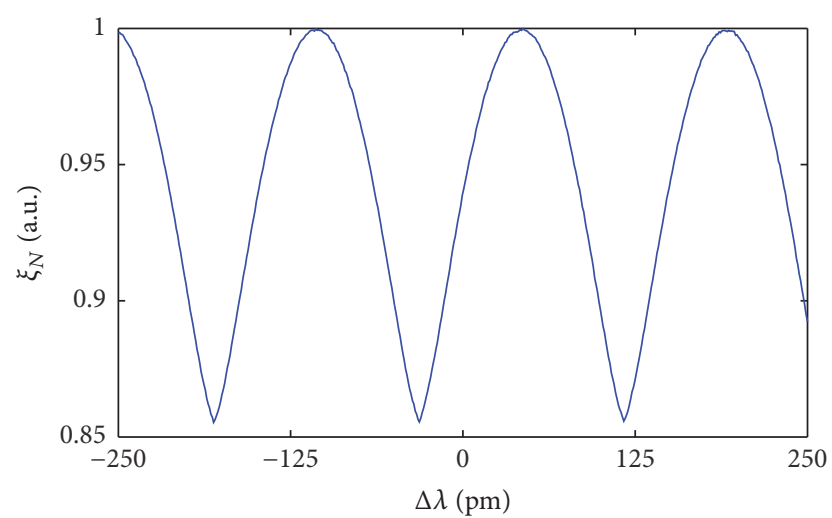

FIGURE 3: High-rank eigenvalue $\xi_{N}$ as a function of wavelength shift $\Delta \lambda$, obtained for FBG simulation in high-SNR conditions (SNR = $60 \mathrm{~dB})$.

In the original implementation of the KLT, the highest rank eigenvalue $\xi_{N}$ is used as a metric to estimate the wavelength shift [44]. Doing this process, it is possible to analyze the relationship between $\xi_{N}$ and the wavelength shift $\Delta \lambda$, as shown in Figure 3. The chart shows that $\xi_{N}$ has a periodic dependence on $\Delta \lambda$, and the period is equal to the wavelength grid (156 pm in this simulation). Therefore, the KLT is capable of obtaining a very accurate FBG tracking, provided that the correct semiperiod of the $\xi_{N}(\Delta \lambda)$ function is identified; this task, as shown in [44], can be performed by using any of the aforementioned techniques such as centroid [36] or Gaussian fitting [35].

3.2. Improved KLT for Small Signal Analysis. In the small signal analysis, we are interested in detecting small wavelength shifts around the FBG central wavelength $\lambda_{B, 0}$. Focusing on small wavelength shift, we can introduce two substantial improvements to the KLT kernel, described by (7)-(11), with the aim of improving the precision sensing features.

In first place, we focus on the FFT operation in (7) and (8). The FFT is effective in spreading the energy of the input signal $\underline{R}$, a peak-shaped function, into a set of $N$ samples, which simplifies the KLT analysis. It is possible to substitute the FFT block with a different spectral estimator, which contributes to noise reduction without an excessive computational complexity. For this task, the Capon power spectral density (PSD) estimator [50, 51], often also referred as the minimum variance estimator [49], is chosen. In this application, the Capon estimator owns a key advantage: rather than returning the spectral estimate on the whole set of digital frequencies, that is, $[0,1 /(N-1), 2 /(N-1), \ldots, 1]$, it allows the user to arbitrarily select the frequency range of analysis.

The Capon estimator, described in [50] and implemented as in [51], is a power spectral density estimator based on a simple concept: for each digital frequency $f_{D}$, the goal is to design a finite impulse response (FIR) bandpass filter with $N$ taps, such that the frequency response of the filter is equal to 1 at the design frequency $f_{D}$, while the variance of the filtered signal is minimized $[49,50]$. Following the original implementation, the PSD of the input signal $\underline{R}$ is given by

$$
\operatorname{PSD}_{\underline{R}}\left(f_{D}\right)=\frac{1}{\underline{a}^{H}\left(f_{D}\right) \underline{R}_{R R}^{-1} \underline{a}\left(f_{D}\right)}
$$

for each digital frequency. In (12), the term $\underline{\underline{R}}_{R R}$ is the estimate of the covariance matrix of $\underline{R}$, defined as in $[50] ; H(\cdot)$ refers to the Hermitian operator. The term $\underline{a}$ is the so-called steering vector, defined as

$$
\underline{a}\left(f_{D}\right)=\left[\begin{array}{c}
1 \\
\exp \left(-j 2 \pi f_{D}\right) \\
\vdots \\
\exp \left(-j 2 \pi(T-1) f_{D}\right)
\end{array}\right] .
$$

The PSD is computed for each $f_{D}$ frequency, arbitrarily selected. Unlike the FFT, with the Capon estimator, it is possible to select the range and the length of the digital frequency axis. In this framework, we select the length $M$ of the frequency axis $\underline{f}_{D}=\left[f_{D, 1}, f_{D, 2}, \ldots, f_{D, M}\right]$ as $M<N$, in order to reduce the computational complexity. In this specific application, the choice is $\underline{f}_{D}=[0.1,0.125,0.15,0.175,0.2]$, focusing on the mid-range of frequencies and setting $M=5$. Other choices that cut off the low-frequency bias yield similar performances, while setting a low value for $M$ contributes to lower the computational complexity. Thus, after computing the $M$-size PSD with Capon estimator, we can rewrite (9):

$$
\begin{aligned}
& \stackrel{M}{=} \\
& =\left[\begin{array}{cccc}
\operatorname{PSD}\left(f_{D, 1}\right) & \operatorname{PSD}\left(f_{D, 2}\right) & \ldots & \operatorname{PSD}\left(f_{D, M}\right) \\
\operatorname{PSD}\left(f_{D, 2}\right) & \operatorname{PSD}\left(f_{D, 1}\right) & \ddots & \vdots \\
\vdots & \ddots & \ddots & \operatorname{PSD}\left(f_{D, 2}\right) \\
\operatorname{PSD}\left(f_{D, M}\right) & \ldots & \operatorname{PSD}\left(f_{D, 2}\right) & \operatorname{PSD}\left(f_{D, 1}\right)
\end{array}\right] .
\end{aligned}
$$

The second modification lies in the choice of the highrank eigenvalue as a metric to estimate the wavelength shift. This choice, initially drawn in [44], was justified by the initial application of Maccone's KLT to the extraction of unknown weak signals buried into noise [47]. However, in the FBG spectrum detection, we need to detect small variations, of a peak signal, close to the quantization limit of the spectrometer, and with a moderate SNR (typically within $30 \mathrm{~dB}$ and $60 \mathrm{~dB}[38,39,44,45])$. Thus, moving away from the original implementation, a possible choice to further improve accuracy is to choose a different eigenvalue as the key metric. While low-rank eigenvalues still withhold most of the noise energy, as demonstrated in $[44,45]$, we can shift our analysis from the highest rank eigenvalue $\left(\xi_{M}\right)$ to the second-rank eigenvalue $\left(\xi_{M-1}\right)$.

Figure 4 justifies the selection of the eigenvalue rank in precision sensing. For two different SNR values $(50 \mathrm{~dB}$ and $60 \mathrm{~dB}$ ), the chart reports the normalized eigenvalues $\xi_{M-1}$, as proposed in this context, and $\xi_{M}$, as originally proposed 


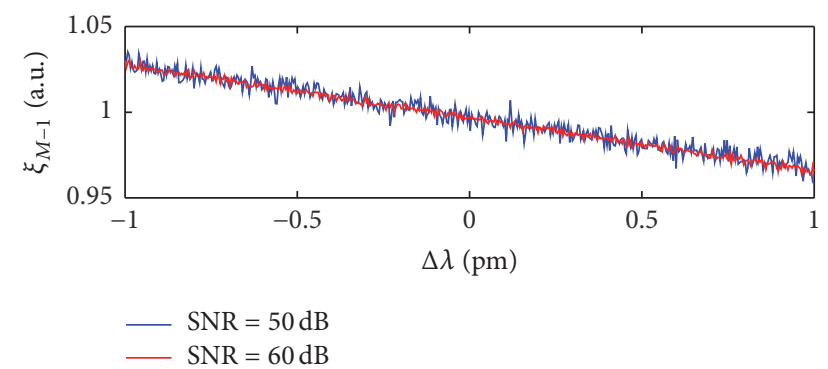

(a)

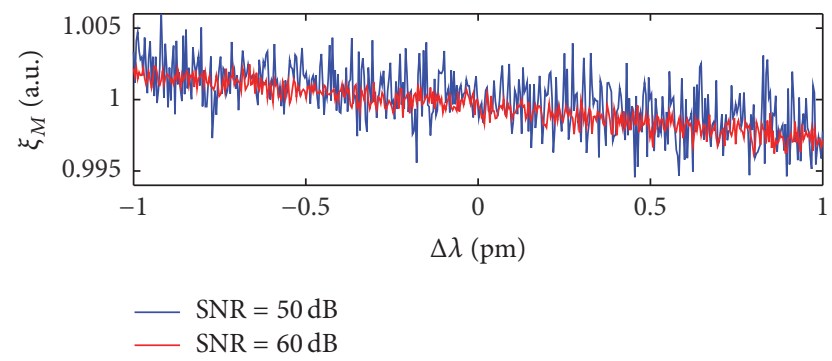

(b)

FIGURE 4: Comparison of different eigenvalue tracking approaches, in small FBG wavelength shift: after applying (9)-(14) for different values of wavelength shift within $\pm 1 \mathrm{pm}$, in different SNR conditions $(50 \mathrm{~dB}$ and $60 \mathrm{~dB})$, the chart displays the highest rank eigenvalue $\xi_{M}$ (with $M=6$ ) on (b) and the second-rank eigenvalue $\xi_{M-1}$ on (a). In both cases, eigenvalues have been normalized by the eigenvalue obtained in reference conditions $(\Delta \lambda=0 \mathrm{pm})$.

in [44]. Data are reported for 500 values of wavelength shift, included within $-1 \mathrm{pm}$ and $+1 \mathrm{pm}$, in order to simulate a small wavelength shift, such as small vibration detection. In order to compare the sensitivity, data have been normalized by the reference value, obtained with no wavelength shift. The figure shows that the new choice of second-rank eigenvalue in the KLT yields a higher resolution: we observe a variation of $3.10 \%$ of $\xi_{M-1}$ for a wavelength shift of $1 \mathrm{pm}$, while with the original algorithm the variation of $\xi_{M}$ is only $0.23 \%$. This increased sensitivity leads to a higher accuracy and is essential for obtaining an accurate detection of small, subpicometer wavelength shifts.

The choice of $\xi_{M-1}$ as the wavelength shift indicator, rather than the highest rank eigenvalue $\xi_{M}$ or the lower rank $\xi_{M-2}, \xi_{M-3}, \ldots$, is in partial agreement with the discussion in [45]. Two factors can be taken into consideration. At first, the problem hereby presented differs from Maccone's KLT formulation [47] since the algorithm does not have to detect a small signal buried into noise but a small shift of a signal clearly laying over the noise threshold and very close to the quantization limit of the spectrometer used as detector. Thus, the algorithm performs better when the values in correspondence of half-maximum of the reflection spectrum of the FBG are tracked, because, in this point, the derivative of $\underline{R}(\underline{\lambda})$ is the maximum. Since the energy of these points is significantly lower than the reflectivity peak, through the Capon estimator, they tend to have strong energy in the second-rank eigenvalues. The second aspect is

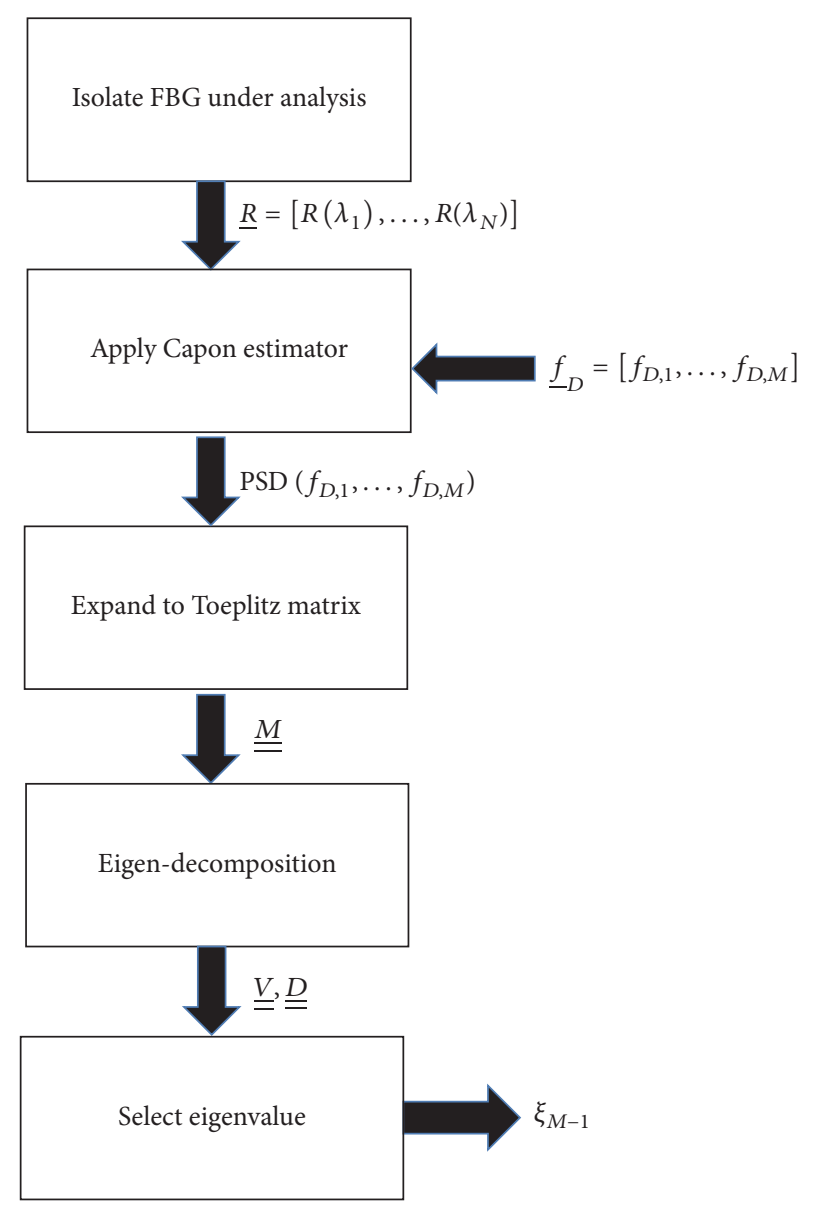

FIGURE 5: Proposed algorithm for small signal decoding of FBGs based on KLT and Capon estimator.

noise, which as shown in [48] tends to be confined in the low-rank eigenvalues, and for this reason it is preferable to avoid choosing $\xi_{M-2}$ or lower rank eigenvalues. In Figure 4, such values are not reported but simulations show that the accuracy is much worse than $\xi_{M}$.

To recap, Figure 5 shows the final implementation of the KLT-based FBG tracking algorithm, for small signal analysis.

\section{Results and Discussion}

In this section, the performance analysis of the FBG decoding method is outlined, using the simulation framework as in Section 3 and the decoding method as in Figure 5.

Figure 6 analyzes the variation of $\xi_{M-1}$ (normalized, as in the Figure 4) in different SNR conditions. In the left column, we observe that the KLT-based algorithm is capable of correctly detecting wavelength shifts in the order of $\pm 5 \mathrm{pm}$, which is the accuracy of several commercial lowcost spectrometers as it appears on datasheets [33, 37]. In the central column, we observe that even variations of $\pm 0.5 \mathrm{pm}$ are detected, with different precision: with high SNR, detection is still very accurate; while lowering the SNR to $45 \mathrm{~dB}$ we start observing the effect of accuracy. Finally, the right column shows the behavior of the KLT algorithm in 

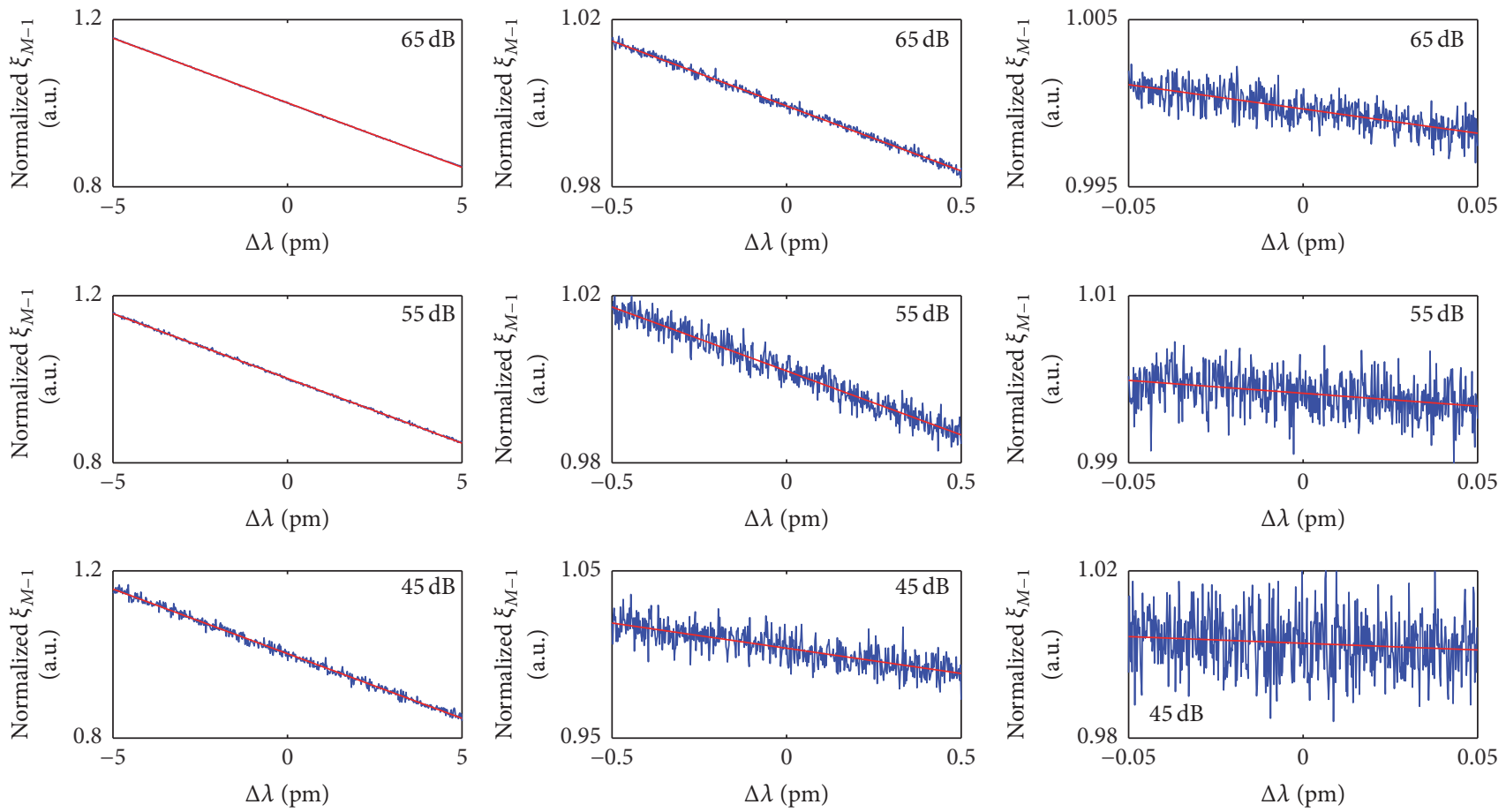

FIGURE 6: Variation of the second-rank eigenvalue $\xi_{M-1}$, normalized as in Figure 4, for different values of SNR ( $65 \mathrm{~dB}, 55 \mathrm{~dB}$, and $\left.45 \mathrm{~dB}\right)$ and different wavelength shift ranges $( \pm 5 \mathrm{pm}, \pm 0.5 \mathrm{pm}$, and $\pm 0.05 \mathrm{pm})$. The charts report both the simulated data (blue) and the linear fit (red).

presence of a small wavelength shift $( \pm 0.05 \mathrm{pm})$. We observe that, while for low SNR the accuracy starts to get insufficient, in high-SNR conditions, we can still obtain a quite clear detection. In Figure 6, we can estimate the sensitivity, defined as the ratio between the variation of normalized $\xi_{M-1}$ and the variation of $\Delta \lambda$, of $-0.0315 \mathrm{pm}^{-1}$.

In this small signal analysis, the root mean square error (RMSE) between the wavelength shift and its estimate obtained with KLT can be evaluated. For SNR $=65 \mathrm{~dB}$, the RMSE is $22.2 \mathrm{fm}$; for SNR $=55 \mathrm{~dB}$, the RMSE is $67.8 \mathrm{fm}$; for $\mathrm{SNR}=45 \mathrm{~dB}$, the RMSE is $221 \mathrm{fm}$. Thus, the algorithm based on KLT excels in identifying small wavelength shifts, and with SNR values typical of several spectrometers, the error is still subpicometer level.

This concept is expanded for the evaluation of the accuracy. In Figure 7, the accuracy as a function of SNR is reported; data are evaluated for different values of $\kappa L$ ranging from 0.3 to 5 , which corresponds to growing values of peak reflectivity and FBG bandwidth, in order to determine how the KLT operates with different types of FBG. In the chart, the accuracy is estimated as the wavelength shift range which withholds $99 \%$ of the wavelength detection error, estimated over 10000 samples for each SNR. We observe that the best performance is obtained for a $99 \%$ reflective grating, whereas the $1 \mathrm{pm}$ accuracy is obtained with $30 \mathrm{~dB}$ SNR, while for $\kappa L=$ 1 the required SNR is $35 \mathrm{~dB}$. Sub-pm accuracy on detection is possible with SNR ranging from $50 \mathrm{~dB}$ to $55 \mathrm{~dB}$, which is compatible with most commercial spectrometers. We observe that performances decrease for very strong grating, whereas small shifts cannot be appreciated, or with poorly reflective FBGs, whereas the quantization noise is detrimental. Making

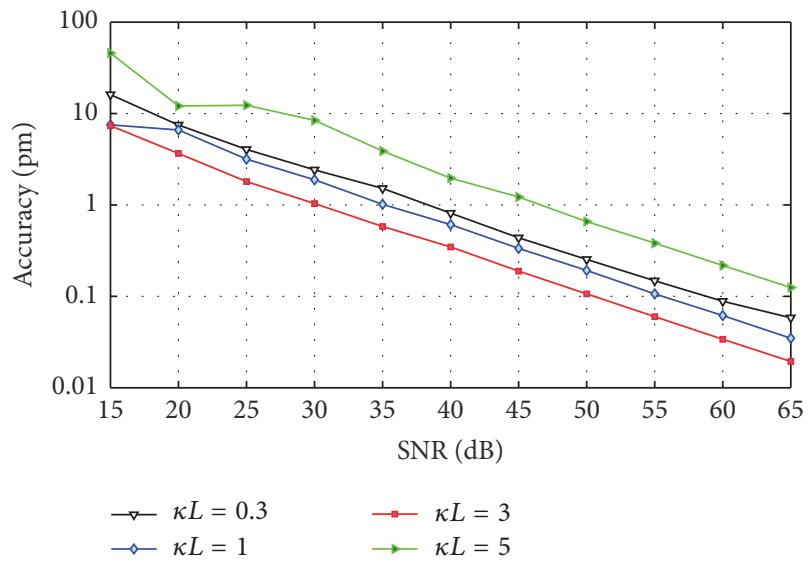

FIGURE 7: Wavelength detection accuracy (99\% interval of confidence) as a function of SNR. Data are reported for different values of $\kappa L$ coefficients: $0.3,1,3$, and 5 (corresponding to peak reflectivity $8.4 \%, 58.0 \%, 99.0 \%$, and $99.98 \%)$.

a linear fit to the data in Figure 7, we estimate that the accuracy follows the following approximate trend:

$$
\text { Accuracy }[\mathrm{pm}] \propto 10^{-0.0475 \times \mathrm{SNR}[\mathrm{dB}]},
$$

which is compatible with [45], although the benchmark for simulation and the definition of SNR is different.

Considering $\kappa L=1$, Figure 8 aims to evaluate the behavior of the KLT algorithm, responding to a $100 \mathrm{~Hz}$ sinusoidal signal, having peak-to-peak amplitude of $2 \mathrm{pm}$; this is a typical study case, representative of dynamic FBG 


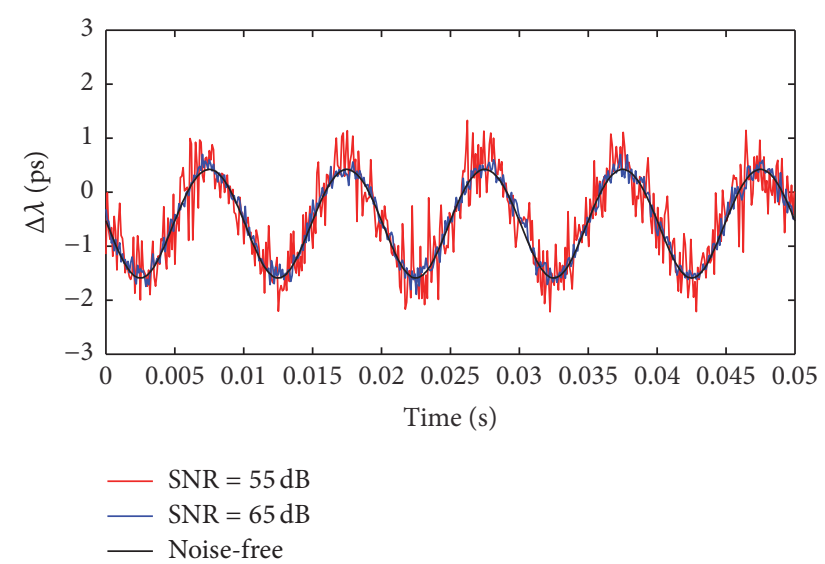

FIgURE 8: Response of the KLT detection algorithm to a $100-\mathrm{Hz}$ sine wave.

TABLE 1: Accuracy and computation time as a function of $M$.

\begin{tabular}{lcc}
\hline$M$ & Accuracy $(\mathrm{pm})$ & Computation time $(\mu \mathrm{s})$ \\
\hline 3 & 0.45 & 108 \\
5 & 0.22 & 126 \\
8 & 0.21 & 145 \\
12 & 0.21 & 186 \\
15 & 0.21 & 209 \\
25 & 0.22 & 334 \\
\hline
\end{tabular}

sensing $[10,12,35]$. It is possible to see that, despite the poor amplitude, below the detection range of most algorithms applied to spectrometers, the sine wave is still detectable, and the precision is sufficient for frequency analysis, as required in structural engineering $[6,7]$.

An important factor for consideration is the computation time and how it relates to the length of the size $M$ of the KLT matrix. Table 1 shows, for different values of $M$, the obtained accuracy for SNR $=50 \mathrm{~dB}$ and the computation time required for one wavelength shift estimate. All data are obtained in MATLAB, on a $2.6 \mathrm{GHz}$ Intel Core i5 processor with $16 \mathrm{~GB}$ RAM memory. It is possible to see that the accuracy is substantially the same for any $M \geq 5$, while only for $M=3$ we observe a decay of performance due to the poorer noise handling. The computation time, estimated over 10000 estimates, is $126 \mu$ s for $M=5$, which is the setting used in previous simulations, and increases for growing values of $M$. Even for $M=25$, the computation time remains well below the original KLT, which is executed in $1 \mathrm{~ms}$ on the same hardware. Such high computation speed is compatible with operation at $5 \mathrm{kHz}$, which is the maximum speed of most spectrometers operating with RS-232 protocol [33].

\section{Experimental Results}

A brief experimental characterization has been set up, with the purpose of validating the prior performance analysis and showing that the KLT is effective in real-case scenarios. The setup in Figure 1 has been implemented experimentally, using

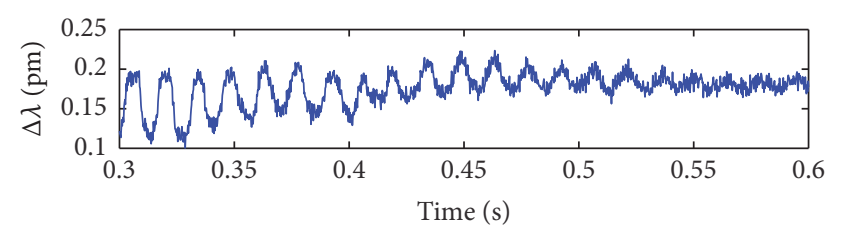

(a)

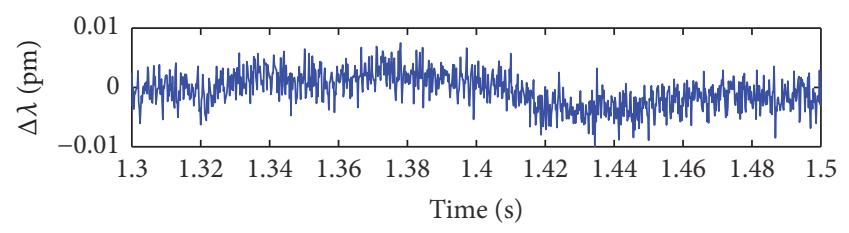

(b)

FIGURE 9: Wavelength shift, estimated in experimental measurements carried out with a commercial spectrometer, operating at $5 \mathrm{kHz}$. (a) shows the response to a $25 \mathrm{~Hz}$ exponentially damped tone, while (b) shows the response in absence of stimuli.

off-the-shelf equipment. The light source is a SLED (Exalos EXS210054-01 with its control board, operating around $1550 \mathrm{~nm}$ ), while the detector is a high-speed spectrometer (Ibsen 512 -USB, $80 \mathrm{~nm}$ operative range, $3 \mathrm{kHz}$ detection speed). The sensor is a $1550-\mathrm{nm}$ FBG with $0.5 \mathrm{~cm}$ length, fabricated on a drawing tower (FBGS International, DTG1550). The FBG has reflectivity $10 \%$. The FBG has been placed on a vibrating support, having resonance frequency $\sim 25 \mathrm{~Hz}$, which has been mechanically excited. In order to reproduce a small signal, the FBG has been placed on the vibrating support with a loose fixing, made with scotch tape. All spectra have been processed as in Figure 5.

Figure 9(a) shows the response of the FBG to a mechanical excitation, which results in an exponentially damped signal. Despite the small amplitude $(0.1 \mathrm{pm})$, the KLT is capable of returning a quite clear waveform, which can be visually detected. Figure 9 (b) shows the response, in absence of excitation: in this case, we see that the noise is extremely limited, with standard deviation equal to $0.032 \mathrm{pm}$.

The results, in line with the previous simulative analysis, confirm that the KLT algorithm is successful in decoding small variations of wavelength shift, well below the picometer level, despite the coarse wavelength sampling. The algorithm for detection is also computationally fast, compatible with structural health monitoring and low-frequency acoustics, as well as static sensing.

\section{Conclusions}

FBGs are strongly increasing their penetration into sensing technologies, and among fiber-optic sensors they are one of the most popular approaches. The FBG market is consolidating and detection hardware is becoming more affordable. Furthermore, recent innovations such as drawtower grating [28], femtosecond laser inscription [30], and FBG into multicore fibers [31] are promoting this approach into frontier sensing applications. 
On the other side, in order to become attractive and competitive with mainstream sensing technologies, it is essential to reduce the cost of sensing equipment. Lowcost spectrometers are becoming more attractive, but they sample the FBG spectra on a coarse wavelength grid, which is unpractical to detect small shifts with precision. Most datasheets mark the wavelength detection accuracy to $\sim 5 \mathrm{pm}$, when spectral sampling is $156 \mathrm{pm}$ (512 pixels over $80 \mathrm{~nm}$ ).

In this paper, an algorithm based on KLT is proposed with the aim of improving the detection of small wavelength shift. The FBG spectrum, as acquired by the spectrometer, is processed using a Capon estimator and then a KLT. As a result, sub-pm wavelength shift can be easily detected, with typical RMSE ranging from 0.02 to $0.2 \mathrm{pm}$, and subpicometer accuracy, depending on the SNR. The algorithm is fast, compatible with $5 \mathrm{kHz}$ operation [33]. A brief experimental validation demonstrates the operation of the algorithm. The proposed algorithm can be easily implemented on software for commercial spectrometers and is particularly suited for precision sensing.

\section{Competing Interests}

The author declares that there is no conflict of interests regarding the publication of this paper.

\section{References}

[1] Y.-J. Rao, "In-fibre Bragg grating sensors," Measurement Science and Technology, vol. 8, no. 4, pp. 355-375, 1997.

[2] A. Othonos and K. Kalli, Fiber Bragg Gratings: Fundamentals and Applications, Artech House, 1999.

[3] A. D. Kersey, M. A. Davis, H. J. Patrick et al., "Fiber grating sensors," Journal of Lightwave Technology, vol. 15, no. 8, pp. 1442-1463, 1997.

[4] T. Erdogan, "Fiber grating spectra," Journal of Lightwave Technology, vol. 15, no. 8, pp. 1277-1294, 1997.

[5] J. Skaar, L. Wang, and T. Erdogan, "On the synthesis of fiber Bragg gratings by layer peeling," IEEE Journal of Quantum Electronics, vol. 37, no. 2, pp. 165-173, 2001.

[6] D. Kinet, P. Mégret, K. W. Goossen, L. Qiu, D. Heider, and C. Caucheteur, "Fiber Bragg grating sensors toward structural health monitoring in composite materials: challenges and solutions," Sensors, vol. 14, no. 4, pp. 7394-7419, 2014.

[7] T. H. T. Chan, L. Yu, H. Y. Tam et al., "Fiber Bragg grating sensors for structural health monitoring of Tsing Ma bridge: background and experimental observation," Engineering Structures, vol. 28, no. 5, pp. 648-659, 2006.

[8] G. C. Kahandawa, J. Epaarachchi, H. Wang, and K. T. Lau, "Use of FBG sensors for SHM in aerospace structures," Photonic Sensors, vol. 2, no. 3, pp. 203-214, 2012.

[9] M. Mieloszyk, L. Skarbek, M. Krawczuk, W. Ostachowicz, and A. Zak, "Application of fibre Bragg grating sensors for structural health monitoring of an adaptive wing," Smart Materials and Structures, vol. 20, no. 12, Article ID 125014, 2011.

[10] Y. R. García, J. M. Corres, and J. Goicoechea, "Vibration detection using optical fiber sensors," Journal of Sensors, vol. 2010, Article ID 936487, 12 pages, 2010.
[11] K. Li, "Review of the strain modulation methods used in Fiber Bragg grating sensors," Journal of Sensors, vol. 2016, Article ID 1284520, 8 pages, 2016.

[12] L. Mohanty, L. M. Koh, and S. C. Tjin, "Fiber Bragg grating microphone system," Applied Physics Letters, vol. 89, no. 16, Article ID 161109, 2006.

[13] M. Vidakovic, I. Armakolas, T. Sun, J. Carlton, and K. T. Grattan, "Fibre Bragg grating-based acoustic sensor array for improved condition monitoring of marine lifting surfaces," Journal of Lightwave Technology, vol. 34, no. 18, pp. 4336-4342, 2016.

[14] A. Laudati, F. Mennella, M. Giordano, G. D’Altrui, C. Calisti Tassini, and A. Cusano, "A fiber-optic Bragg grating seismic sensor," IEEE Photonics Technology Letters, vol. 19, no. 24, pp. 1991-1993, 2007.

[15] Z.-F. Wang, J. Wang, Q.-M. Sui et al., "Development and application of smart geogrid embedded with fiber Bragg grating sensors," Journal of Sensors, vol. 2015, Article ID 108209, 10 pages, 2015.

[16] V. Mishra, N. Singh, U. Tiwari, and P. Kapur, "Fiber grating sensors in medicine: current and emerging applications," Sensors and Actuators A: Physical, vol. 167, no. 2, pp. 279-290, 2011.

[17] D. J. Webb, S. Jones, L. Zhang, I. Bennion, M. W. Hathaway, and D. A. Jackson, "First in-vivo trials of a fiber Bragg grating based temperature profiling system," Journal of Biomedical Optics, vol. 5, no. 1, pp. 45-50, 2000.

[18] L. Dziuda, F. W. Skibniewski, M. Krej, and J. Lewandowski, "Monitoring respiration and cardiac activity using fiber Bragg grating-based sensor," IEEE Transactions on Biomedical Engineering, vol. 59, no. 7, pp. 1934-1942, 2012.

[19] J. W. Arkwright, N. G. Blenman, I. D. Underhill et al., "Measurement of muscular activity associated with peristalsis in the human gut using fiber Bragg grating arrays," IEEE Sensors Journal, vol. 12, no. 1, pp. 113-117, 2012.

[20] L. Mohanty, S. C. Tjin, D. T. T. Lie, S. E. C. Panganiban, and P. K. H. Chow, "Fiber grating sensor for pressure mapping during total knee arthroplasty," Sensors and Actuators A: Physical, vol. 135, no. 2, pp. 323-328, 2007.

[21] A. F. Fernandez, A. I. Gusarov, B. Brichard et al., "Temperature monitoring of nuclear reactor cores with multiplexed fiber Bragg grating sensors," Optical Engineering, vol. 41, no. 6, pp. 1246-1254, 2002.

[22] S. J. Mihailov, "Fiber bragg grating sensors for harsh environments," Sensors, vol. 12, no. 2, pp. 1898-1918, 2012.

[23] F. Marignetti, E. de Santis, S. Avino et al., "Fiber Bragg grating sensor for electric field measurement in the end windings of high-voltage electric machines," IEEE Transactions on Industrial Electronics, vol. 63, no. 5, pp. 2796-2802, 2016.

[24] C. Ambrosino, S. Campopiano, A. Cutolo, and A. Cusano, "Sensitivity tuning in terfenol-D based fiber bragg grating magnetic sensors," IEEE Sensors Journal, vol. 8, no. 9, pp. 15191520, 2008.

[25] M. Buric, T. Chen, M. Maklad, P. R. Swinehart, and K. P. Chen, "Multiplexable low-temperature fiber Bragg grating hydrogen sensors," IEEE Photonics Technology Letters, vol. 21, no. 21, pp. 1594-1596, 2009.

[26] K. O. Hill and G. Meltz, "Fiber Bragg grating technology fundamentals and overview," Journal of Lightwave Technology, vol. 15, no. 8, pp. 1263-1276, 1997.

[27] K. O. Hill, B. Malo, F. Bilodeau, D. C. Johnson, and J. Albert, "Bragg gratings fabricated in monomode photosensitive optical fiber by UV exposure through a phase mask," Applied Physics Letters, vol. 62, pp. 1035-1037, 1993. 
[28] E. Lindner, A. Hartung, D. Hoh et al., "Trends and future of fiber Bragg grating sensing technologies: tailored draw tower gratings (DTGs)," in Optical Sensing and Detection III, vol. 9141 of Proceedings of SPIE, May 2014.

[29] FBGS, "FBG applications," http://www.fbgs.com/.

[30] T. Geernaert, K. Kalli, C. Koutsides et al., "Point-by-point fiber bragg grating inscription in free-standing step-index and photonic crystal fibers using near-IR femtosecond laser," Optics Letters, vol. 35, no. 10, pp. 1647-1649, 2010.

[31] M. Becker, T. Elsmann, A. Lorenz et al., "Fiber Bragg grating inscription in optical multicore fibers," in 24th International Conference on Optical Fibre Sensors, vol. 9634 of Proceedings of SPIE, Curitiba, Brazil, September 2015.

[32] N. Liu, Y. Li, H. Wang, W. Chen, and P. Lu, "Directional bend sensing with bragg gratings in all solid bragg fibers," IEEE Photonics Technology Letters, vol. 23, no. 17, pp. 1237-1239, 2011.

[33] Ibsen Photonics, Interrogation Monitors, http://www.ibsenphotonics.com.

[34] A. Ezbiri, S. E. Kanellopoulos, and V. A. Handerek, "High resolution instrumentation system for fibre-Bragg grating aerospace sensors," Optics Communications, vol. 150, no. 1-6, pp. 43-48, 1998.

[35] W. Chen, A. Vallan, and G. Perrone, "Performance assessment of a fast temperature sensing system based on bare FBGs and fast spectrum analyzer," in Proceedings of the 1st International Electronic Conference on Sensors and Applications, vol. 1 of Sciforum Electronic Conference Series, pp. 1-16, June 2014.

[36] C. G. Atkins, M. A. Putnam, and E. J. Friebele, "Instrumentation for interrogating many-element fiber Bragg grating arrays," in Proceedings of the Smart Structures and Materials 1995: Smart Sensing, Processing, and Instrumentation, Proceedings of SPIE, pp. 257-267, San Diego, Calif, USA, February 1995.

[37] Micron Optics, Instruments, http://www.micronoptics.com.

[38] Bayspec, "FBG Interrogation Analyzer," http://www.bayspec .com.

[39] National-Instruments, "FBG applications," http://www.ni.com/ opticalsensing/.

[40] J. M. Gong, C. C. Chan, W. Jin, J. M. K. MacAlpine, M. Zhang, and Y. B. Liao, "Enhancement of wavelength detection accuracy in fiber Bragg grating sensors by using a spectrum correlation technique," Optics Communications, vol. 212, no. 1-3, pp. 29-33, 2002.

[41] C. Caucheteur, K. Chah, F. Lhommé, M. Blondel, and P. Mégret, "Autocorrelation demodulation technique for fiber Bragg grating sensor," IEEE Photonics Technology Letters, vol. 16, no. 10, pp. 2320-2322, 2004.

[42] A. Lamberti, S. Vanlanduit, B. De Pauw, and F. Berghmans, "A novel fast phase correlation algorithm for peak wavelength detection of fiber Bragg grating sensors," Optics Express, vol. 22, no. 6, pp. 7099-7112, 2014.

[43] Y. Hu, W. Mo, K. Dong, F. Jin, and J. Song, "Using maximum spectrum of continuous wavelet transform for demodulation of an overlapped spectrum in a fiber Bragg grating sensor network," Applied Optics, vol. 55, no. 17, pp. 4670-4675, 2016.

[44] D. Tosi, "KLT-based algorithm for sub-picometer accurate FBG tracking with coarse wavelength sampling," IEEE Photonics Technology Letters, vol. 27, no. 20, pp. 2134-2137, 2015.

[45] D. Tosi, "Advanced interrogation of fiber-optic Bragg grating and Fabry-Perot sensors with KLT analysis," Sensors, vol. 15, no. 11, pp. 27470-27492, 2015.
[46] C. Maccone, Telecommunications, KLT and Relativity, IPI Press, 1994.

[47] C. Maccone, "The KLT (Karhunen-Loève Transform) to extend SETI searches to broad-band and extremely feeble signals," Acta Astronautica, vol. 67, no. 11-12, pp. 1427-1439, 2010.

[48] C. Maccone, "Advantages of Karhunen-Loève transform over fast Fourier transform for planetary radar and space debris detection," Acta Astronautica, vol. 60, no. 8-9, pp. 775-779, 2007.

[49] A. Zaknich, Principles of Adaptive Filters and Self-Learning Systems, Springer, Berlin, Germany, 2006.

[50] J. Capon, "High-resolution frequency-wavenumber spectrum analysis," Proceedings of the IEEE, vol. 57, no. 8, pp. 1408-1418, 1969.

[51] J. Benesty, J. Chen, and Y. Huang, "Recursive and fast recursive Capon spectral estimators," EURASIP Journal on Advances in Signal Processing, vol. 2007, Article ID 045194, 2007. 


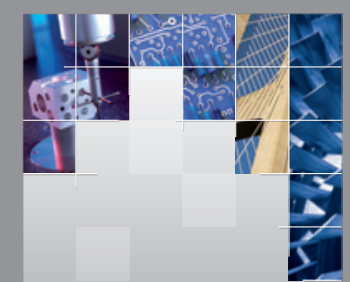

\section{Enfincering}
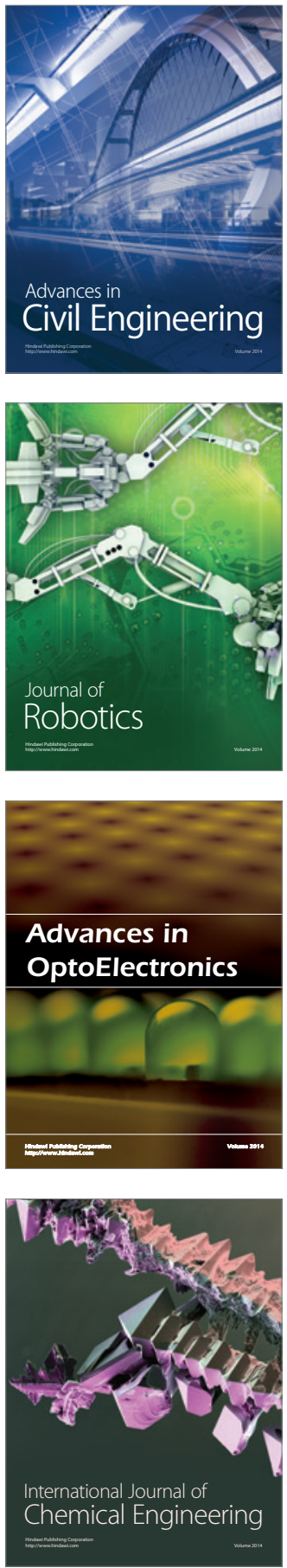

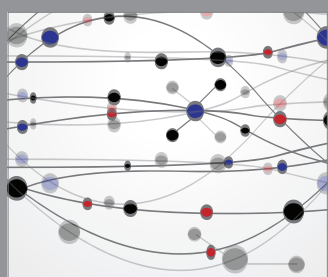

The Scientific World Journal

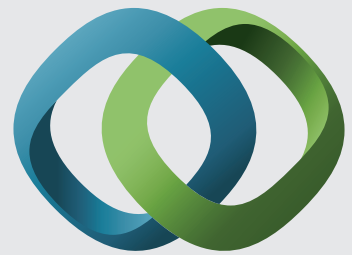

\section{Hindawi}

Submit your manuscripts at

https://www.hindawi.com
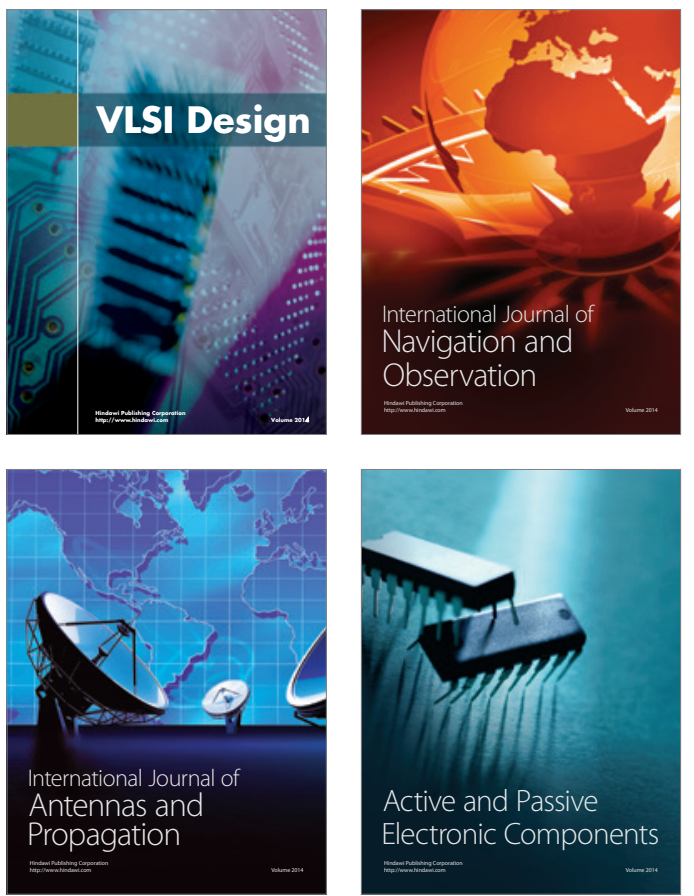
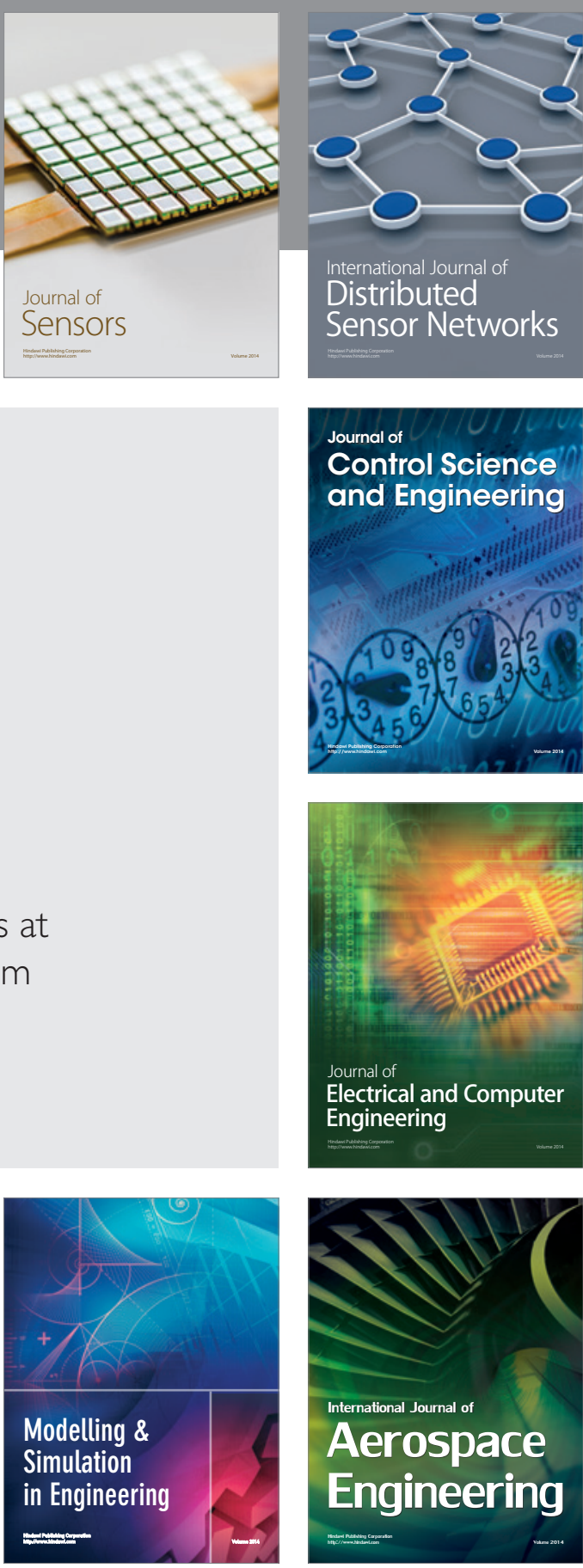

International Journal of

Distributed

Sensor Networks

$-$

Joumal of

Control Science

and Engineering
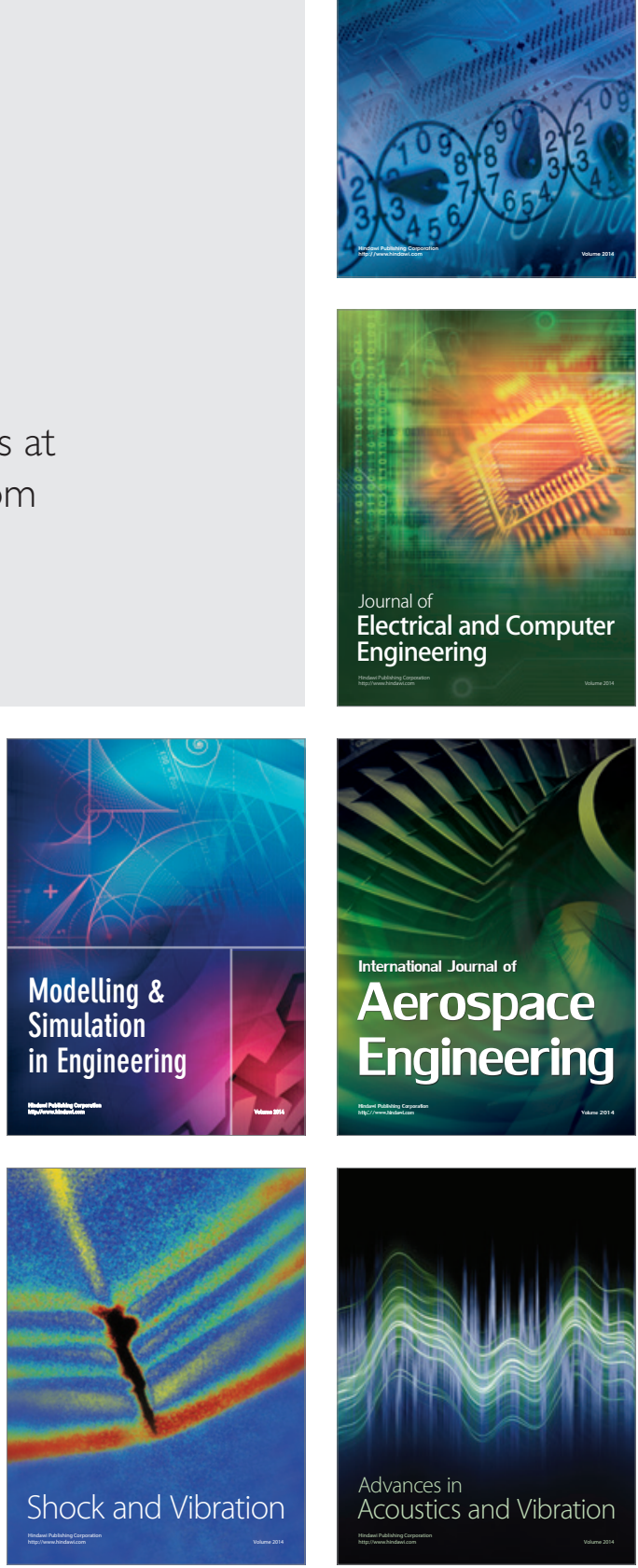